\title{
(2) OPEN ACCESS \\ Is cardiac involvement prevalent in highly trained athletes after SARS-CoV-2 infection? A cardiac magnetic resonance study using sex-matched and age-matched controls
}

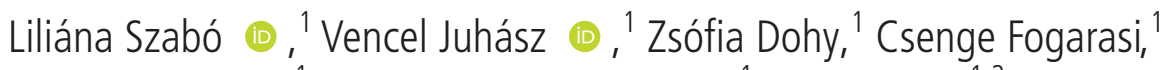

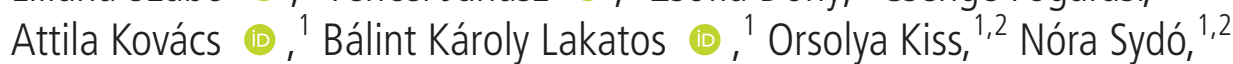 \\ Emese Csulak, ${ }^{1}$ Ferenc Imre Suhai, ${ }^{1}$ Kristóf Hirschberg, ${ }^{1}$ Dávid Becker, ${ }^{1}$ Béla Merkely, ${ }^{1,2}$ \\ Hajnalka Vágó (1) 1,2
}

\begin{abstract}
- Additional supplemental material is published online only. To view, please visit the journal online (http://dx.doi. org/10.1136/bjsports-2021 104576).
\end{abstract}

${ }^{1}$ Heart and Vascular Center, Semmelweis University, Budapest, Hungary

${ }^{2}$ Department of Sports Medicine, Semmelweis University,

Budapest, Hungary

Correspondence to

Dr Hajnalka Vágó, Heart and Vascular Center, Semmelweis University, Budapest, Hungary; vagoha@gmail.com

$\mathrm{BM}$ and $\mathrm{HV}$ contributed equally.

Accepted 10 November 2021

Check for updates

(c) Author(s) (or their employer(s)) 2021. Re-use permitted under CC BY. Published by BMJ.

To cite: Szabó L,

Juhász V, Dohy Z, et al.

Br I Sports Med Epub ahead

of print: [please include Day

Month Year]. doi:10.1136/

bjsports-2021-104576

\section{ABSTRACT}

Objectives To investigate the cardiovascular consequences of SARS-CoV-2 infection in highly trained, otherwise healthy athletes using cardiac magnetic resonance (CMR) imaging and to compare our results with sex-matched and age-matched athletes and less active controls.

Methods SARS-CoV-2 infection was diagnosed by PCR on swab tests or serum immunoglobulin $\mathrm{G}$ antibody tests prior to a comprehensive CMR examination. The CMR protocol contained sequences to assess structural, functional and tissue-specific data.

Results One hundred forty-seven athletes (94 male, median 23, IQR 20-28 years) after SARS-CoV-2 infection were included. Overall, $4.7 \%(n=7)$ of the athletes had alterations in their CMR as follows: late gadolinium enhancement (LGE) showing a non-ischaemic pattern with or without $T 2$ elevation $(n=3)$, slightly elevated native $T 1$ values with or without elevated $\mathrm{T} 2$ values without pathological LGE $(n=3)$ and pericardial involvement $(n=1)$. Only two (1.4\%) athletes presented with definite signs of myocarditis. We found pronounced sport adaptation in both athletes after SARS-CoV-2 infection and athlete controls. There was no difference between CMR parameters, including native $\mathrm{T} 1$ and $\mathrm{T} 2$ mapping, between athletes after SARS-CoV-2 infection and the matched athletic groups. Comparing athletes with different symptom severities showed that athletes with moderate symptoms had slightly greater T1 values than athletes with asymptomatic and mildly symptomatic infections $(p<0.05)$. However, T1 mapping values remained below the cut-off point for most patients. Conclusion Among 147 highly trained athletes after SARS-CoV-2 infection, cardiac involvement on CMR showed a modest frequency $(4.7 \%)$, with definite signs of myocarditis present in only $1.4 \%$. Comparing athletes after SARS-CoV-2 infection and healthy sex-matched and age-matched athletes showed no difference between CMR parameters, including native T1 and T2 values.

\section{INTRODUCTION}

The presence and extent of cardiac involvement in patients with COVID-19 are of great interest, especially among highly trained athletes returning to extreme physical activity after the infection. Emerging yet conflicting evidence has led to greater interest in cardiac magnetic resonance (CMR) imaging studies due to its ability to provide tissuespecific information non-invasively. A cohort study by Puntmann et al ${ }^{1}$ using late gadolinium enhancement (LGE) and novel T1 and T2 mapping sequences showed myocardial involvement in an alarming $78 \%$ of middle-aged patients, raising serious concerns regarding their cardiac health. Approximately one-third of the alterations were solely based on mapping elevations; however, the exact diagnostic and prognostic impact of these contemporary techniques is less well understood than that of widely used techniques such as LGE. ${ }^{2}$

Recently published studies have evaluated cardiac involvement by CMR imaging in athletes who recovered after SARS-CoV-2 infection. Earlier data by Rajpal et $a l^{3}$ and Brito et $a l^{4}$ found a high prevalence of myocardial (15\%) and pericardial (39.5\%) inflammatory alterations among college athletes following SARS-CoV-2 infection. Subsequent publications reported a lower prevalence of cardiac involvement ranging from $0.7 \%$ to $3.0 \%$ in college athletes after SARS-CoV-2 infection. ${ }^{5-7}$

The most recent expert consensus statements regarding the screening of potential cardiac involvement in competitive athletes recovering from SARS-CoV-2 infection highlight the need for more robust data with the inclusion of appropriate control subjects. ${ }^{8}{ }^{9}$ Therefore, our study aimed to investigate cardiac involvement after SARS-CoV-2 infection in young competitive athletes using a comprehensive CMR imaging study, including tissue characterisation and feature-tracking strain analysis. We compared our results with those from healthy sex-matched and age-matched athletes and healthy sex-matched and age-matched less active controls.

\section{METHODS}

Study population

All athletes recovering from SARS-CoV-2 infection who were referred to our centre for CMR examination between July 2020 and February 2021 were consecutively included in this observational study (figure 1). SARS-CoV-2 infection was diagnosed by PCR on swab tests or by serum IgG antibody tests prior to CMR examination. We excluded athletes 


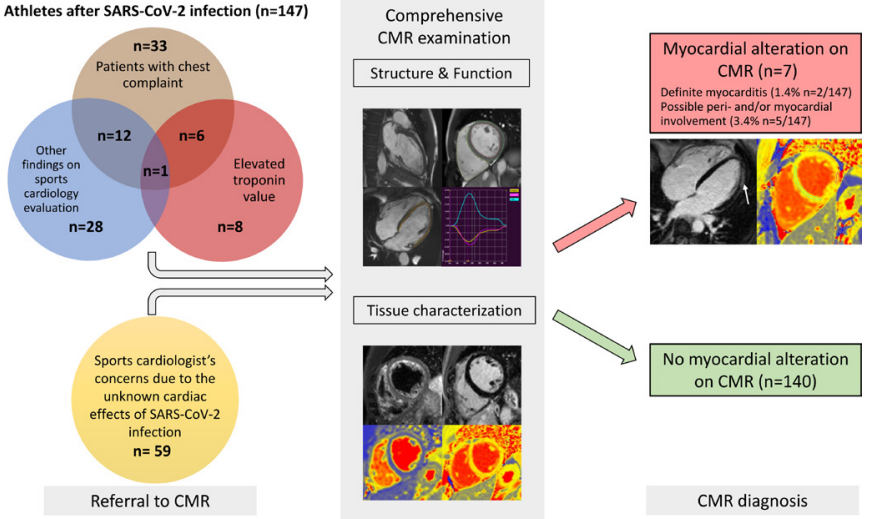

Figure 1 Central illustration. Athletes were referred for CMR by their cardiologists to evaluate the possible structural alterations caused by SARS-CoV-2 infection. CMR referral is summarised as follows: patients who had chest complaints (brown bubble), including chest pain, dyspnoea and palpitation; second, patients who had CMR due to elevated troponin levels (red bubble) with or without accompanying symptoms; third, due to other findings on sports cardiology evaluation (blue bubble) such as alterations on echocardiography and/or 12-leadECG examination; lastly, those referred to CMR due to the unknown cardiac effects of the infection (yellow bubble) despite having negative results on cardiology examination. All athletes underwent a comprehensive CMR examination that contained sequences to assess structural, functional (long-axis and short-axis cine images) and tissuespecific data (T2-weighted images, LGE, native T2 and T1 mappings). Overall, we found cardiac involvement on CMR in only seven patients. Only two presented with definite signs of myocarditis (red box, underneath white arrow showing subepicardial LGE). The majority of athletes had no alterations on their CMR (green box). CMR, cardiac magnetic resonance; $L G E$, late gadolinium enhancement.

(1) aged $<16$ years and (2) performing $<6$ hours of training/ week. Athletes were referred for CMR by their cardiologist to evaluate for possible structural alterations caused by SARSCoV-2 infection, in most cases prior to their return to high levels of sports activity. Detailed information regarding patient referral to CMR is included in figure 1 . All athletes completed a sports-specific questionnaire and a questionnaire regarding their SARS-CoV-2-related symptoms. Symptoms were assessed using the COVID-19 treatment guidelines published by the National Institutes of Health. ${ }^{10}$ Asymptomatic SARS-CoV-2 infection was defined for individuals who tested positive for SARS-CoV-2 and had no symptoms consistent with COVID-19. Mild symptoms were defined as symptoms such as fever, cough, headache, loss of smell and/or taste but not more alarming signs, such as chest pain, dyspnoea and shortness of breath, which were categorised as moderate symptoms. Long-COVID-19 symptoms were persistent symptoms, mostly fatigue and palpitations, extending beyond 4 weeks after the initial infection. Data from the first 12 athletes with post-COVID-19 scanned in our institute published in Journal of the American College of Cardiology (JACC) imaging are incorporated in the current publication. ${ }^{11}$

Clinical data, including 12-lead ECG and high-sensitivity troponin $\mathrm{T}$ (hs $\mathrm{TnT}$ ) were recorded a median of 1 day (0-7 days) prior to the CMR examination. The local laboratory cut-off value for detectable hsTnT was $>2.99 \mathrm{ng} / \mathrm{L}$ and that for elevated hsTnT was $>13.99 \mathrm{ng} / \mathrm{L}$. All examinations were performed after an appropriate quarantine period (10 days).

CMR parameters were compared with those of sex-matched and age-matched healthy athletes $(n=59)$ and healthy, less active controls $(n=56)$. All healthy controls were scanned to establish normal values for the less active and athletic population without any suspicion of cardiovascular pathology prior to the COVID-19 pandemic (59\%) or in athletes and volunteers who tested seronegative for the disease (41\%). Athletes after SARSCoV-2 infection and healthy control athletes both performed high levels of sport activity, the majority of them being professional athletes competing at national or international levels in mixed or endurance sports disciplines (table 1). ${ }^{12}$ Healthy, less active controls performed $<6$ hours of sports activity/week. None of the participants reported a history of cardiovascular disease or consumption of illegal drugs. None of the athletes with post-COVID-19 received steroids during their illness.

\section{CMR protocol}

CMR examinations were performed on a $1.5 \mathrm{~T}$ MRI scanner (Magnetom Aera; Siemens Healthcare, Erlangen, Germany). A comprehensive CMR protocol was carried out, including cine movies, T2-weighted spectral presaturation with inversion recovery, T2 mapping using T2-prep balanced steady-state free precession (b-SSFP), T1 mapping using long-T1 5(3)3 and short-T1 5(3)3 modified look-locker inversion recovery and LGE imaging. Functional imaging was performed using b-SSFP cine sequences in four-chamber, two-chamber and three-chamber long-axis views and a short-axis (SA) stack from the cardiac base to apex with full coverage of the left ventricle (LV) and th right ventricle (RV). Overall, 139 athletes after SARS-CoV-2 infection and 15 healthy control athletes agreed to receive contrast agent. None of the healthy, less active controls were given contrast material. LGE images were acquired using a segmented inversion recovery sequence $10-15 \mathrm{~min}$ after the administration of an intravenous bolus of $0.15 \mathrm{mmol} / \mathrm{kg}$ gadolinium-based contrast agent gadobutrol (Gadovist, Bayer-Schering Pharma) at a rate of $2-3 \mathrm{~mL} / \mathrm{s}$ through an antecubital intravenous line. The inversion time was adjusted to provide optimal suppression of normal myocardium.

\section{Image analysis}

All postprocessing analyses were performed using Medis Suite Software (Medis Medical Imaging Software, The Netherlands). LV and RV volumes, function and mass were calculated from the SA stack using artificial intelligence-based automated contour detection (autoQ application) with manual adjustments if required. Myocardial native T1 and T2 relaxation times were measured conservatively in the midventricular or basal septum (if the midventricular images were technically inadequate for analysis) of the myocardium using motion-corrected images ${ }^{13}$ by an experienced observer blinded to the clinical data and group of a given subject. In case of suspicion of focal T1 mapping elevation, a separate region of interest in that area was drawn. Quantitative deformation assessment was obtained using cine images and analysed using the QStrain application. Global strain values, including LV longitudinal (global longitudinal strain (GLS)), circumferential, radial and RV longitudinal, and free wall strain, were measured. The interpretation of LGE was standardised as follows: myocardial and pericardial LGE was visually defined by two observers based on the presence and pattern. All images were visually assessed by two observers blinded to the clinical data of a given subject. In case of disagreement between the observers, a third CMR specialist with an European Association of Cardiovascular Imaging level 3 certificate was consulted for consensus. Non-ischaemic LGE was defined as midmyocardial and/or subepicardial myocardial LGE confirmed in two 
Table 1 Comparison between athletes after SARS-CoV-2 infection, healthy athlete controls and healthy, less active controls

\begin{tabular}{|c|c|c|c|c|c|}
\hline & $\begin{array}{l}\text { Athletes after SARS- } \\
\text { CoV-2 infection }(n=147)\end{array}$ & $\begin{array}{l}\text { Healthy athletic } \\
\text { controls }(n=59)\end{array}$ & $\begin{array}{l}\text { Healthy, less active } \\
\text { controls }(n=56)\end{array}$ & $\begin{array}{l}\text { Athletes after SARS-CoV-2 } \\
\text { infection versus healthy } \\
\text { athletic controls } \\
P \text { values }\end{array}$ & $\begin{array}{l}\text { Athletes after SARS-CoV-2 } \\
\text { infection versus healthy, less } \\
\text { active controls } \\
P \text { values }\end{array}$ \\
\hline \multicolumn{6}{|l|}{ Group characteristics } \\
\hline Age (years), median (IQR) & $23(20-28)$ & $25(21-29)$ & $24(23-28)$ & 0.146 & 0.062 \\
\hline Sex: female, N (\%) & $53(36)$ & $20(34)$ & $20(36)$ & 0.771 & 0.864 \\
\hline Body surface area $\left(\mathrm{m}^{2}\right)$, average \pm SD & $2 \pm 0.2$ & $2 \pm 0.3$ & $1.9 \pm 0.2$ & 0.413 & 0.003 \\
\hline Heart rate (beats/min), median (IQR) & $60(53-69)$ & $62(56-72)$ & $71(63-84)$ & 0.032 & $<0.001$ \\
\hline $\begin{array}{l}\text { Degree of training (hours/week), } \\
\text { median (IQRS) }\end{array}$ & $15(12-22)$ & $19(15-22)$ & & 0.024 & \\
\hline Sport discipline, N (\%) & & & & 0.077 & \\
\hline Skill & $2(1)$ & $0(0)$ & & & \\
\hline Power & $9(6)$ & $9(15)$ & & & \\
\hline Mixed & $108(74)$ & $35(60)$ & & & \\
\hline Endurance & $28(19)$ & $15(25)$ & & & \\
\hline Member of a national team, $\mathrm{N}(\%)$ & $87(60)$ & $52(91)$ & & $<0.001$ & \\
\hline Member of an Olympic team, N (\%) & $17(12)$ & $15(26)$ & & 0.014 & \\
\hline \multicolumn{6}{|l|}{ CMR parameters } \\
\hline \multicolumn{6}{|c|}{ Standard left and right ventricular parameters } \\
\hline LVEF (\%), median (IQR) & $57(54-60)$ & $56(53-60)$ & $59(57-62)$ & 0.473 & $<0.001$ \\
\hline LVEDVi $\left(\mathrm{mL} / \mathrm{m}^{2}\right)$, median (IQR) & $111(100-123)$ & $111(102-122)$ & $91(83-100)$ & 0.523 & $<0.001$ \\
\hline LVESVi (mL/m²), median (IQR) & $48(40-55)$ & $47(43-53)$ & $38(34-42)$ & 0.52 & $<0.001$ \\
\hline LVSVi $\left(\mathrm{mL} / \mathrm{m}^{2}\right)$, median (IQR) & $63(58-69)$ & $64(58-68)$ & $54(50-59)$ & 0.685 & $<0.001$ \\
\hline LVMi $\left(\mathrm{g} / \mathrm{m}^{2}\right)$ median (IQR) & $58(49-65)$ & $59(50-73)$ & $47(39-51)$ & 0.199 & $<0.001$ \\
\hline RVEF (\%), median (IQR) & $56(53-59)$ & $55(52-58)$ & $57(54-61)$ & 0.14 & 0.014 \\
\hline RVEDVi $\left(\mathrm{mL} / \mathrm{m}^{2}\right)$, median (IQR) & $110(99-121)$ & $113(103-127)$ & $90(79-103)$ & 0.119 & $<0.001$ \\
\hline RVESVi $\left(\mathrm{mL} / \mathrm{m}^{2}\right)$, median (IQR) & $48(41-55)$ & $50(44-59)$ & $38(33-47)$ & 0.055 & $<0.001$ \\
\hline RVSVi (mL/m²), median (IQR) & $61(56-67)$ & $63(57-68)$ & $53(47-58)$ & 0.229 & $<0.001$ \\
\hline \multicolumn{6}{|l|}{ Global left and right ventricular strain } \\
\hline LV-GLS (\%) median (IQR) & $-21(-23$ to -19$)$ & $-20(-23$ to 19$)$ & $-22(-24$ to -20$)$ & 0.942 & $<0.001$ \\
\hline LV-GCS (\%), average \pm SD & $-28 \pm 4$ & $-28 \pm 4$ & $-31 \pm 3$ & 0.426 & $<0.001$ \\
\hline LV-GRS (\%), median (IQR) & $52(46-60)$ & $50(45-58)$ & $56(53-62)$ & 0.609 & $<0.001$ \\
\hline RV-GLS (\%), average \pm SD & $-24 \pm 4$ & $-24 \pm 3$ & $-25 \pm 4$ & 0.691 & 0.21 \\
\hline \multicolumn{6}{|l|}{ Parametric mapping } \\
\hline T1 mapping (ms), median (IQR) & $958(939-970)$ & $955(934-973)$ & $972(960-987)$ & 0.564 & $<0.001$ \\
\hline T2 mapping (ms), median (IQR) & $45(43-46)$ & $44(43-46)$ & $44(43-45)$ & 0.196 & 0.215 \\
\hline
\end{tabular}

CMR, cardiac magnetic resonance; GCS, global circumferential strain; GLS, global longitudinal strain; GRS, global radial strain; LEDVi, left ventricular end diastolic volume index; LV, left ventricular; LVEF, left ventricular ejection fraction; LVESVi, left ventricular end systolic volume index; LVMi, left ventricular mass index; RV, right ventricular; RVEDVi, right ventricular end diastolic volume index; RVEF, right ventricular ejection fraction; RVESVi, right ventricular end systolic volume index; RVMi, right ventricular mass index; SLVi, left ventricular stroke volume index.

perpendicular views. Pericardial involvement was reported if the pericardium showed definite LGE and the thickness of the pericardium was $>2 \mathrm{~mm}$ regardless of pericardial oedema. Hinge point fibrosis was defined as a small volume of focal LGE confined to the inferoseptal segment, where the RV attaches to the septum. We classified cardiac involvement as definite in case of CMR T1 abnormality or LGE showing pathological pattern and CMR T2 abnormality and one or more supporting findings such as decreased LV ejection fraction or elevated troponin level. Possible pericardial/myocardial involvement was reported when we found (1) mild CMR T1 abnormality or the presence of LGE with normal T2, or (2) mildly elevated T1 and T2 mapping with no LGE or other supporting findings.

\section{Follow-up}

We performed midterm follow-up using the institutional electronic database for the patients who returned to our clinic, and we contacted the other athletes via telephone. Athletes completed a questionnaire regarding any ongoing symptoms, their ability to return to high sports activity levels, and their overall experience during the CMR examination. We offered a follow-up cardiological examination, including a CMR scan at our institution, to all athletes reporting reinfection with SARS-CoV-2. All athletes with definite or possible myocardial alteration on their baseline scan were contacted and offered a follow-up CMR examination.

\section{Data management and statistical analysis}

The Shapiro-Wilk test was applied to test the normality of our data. Continuous variables showing a normal distribution are presented as the mean and SD, and those showing a non-normal distribution are reported as medians and IQRs. Categorical variables are presented as frequencies and percentages. Comparisons between participant groups were conducted using independent samples t-tests and Mann-Whitney U tests as appropriate. Nonnormal continuous variables were compared by the KruskalWallis test. $\chi^{2}$ tests were applied to compare the distributions 
of categorical data. Associations were assessed using Spearman's rank correlation analyses. Probability values were two-sided, and $\mathrm{p}$ values of $<0.05$ were considered significant. Elevated T1 and T2 values were defined based on the sequence-specific cut-offs of 2 SDs above the respective means of the healthy, sex-matched and age-matched athlete controls (male athletes: T1: $986 \mathrm{~ms}$, T2: 46 ms; female athletes: T1: $1001 \mathrm{~ms}, \mathrm{~T} 2: 49 \mathrm{~ms})$. MedCalc software V.18.11 (Belgium) and RStudio V.1.3.1.093 (RFoundation, Austria) were used for statistical analysis and graph generation. All data are available on reasonable request.

\section{RESULTS}

Overall, 147 (94 male, median 23, IQR 20-28 years) athletes with prior SARS-CoV-2 infection were included in our study. They were asymptomatic $(n=19)$ or experienced mild $(n=80)$, moderate $(n=43)$ or long-COVID-19 $(n=5)$ symptoms, and none of them required hospital treatment. CMR imaging was performed at a median of 32 days after a positive PCR test. Overall, 4.7\% $(n=7)$ of patients had alterations in their CMR scans, and none of these athletes were asymptomatic. The CMR findings were as follows: LGE showing a non-ischaemic pattern and elevated native T1 mapping consistent with acute myocarditis as per the Lake Louise criteria $(n=1)$; LGE showing a nonischaemic pattern consistent with previous myocarditis with only mildly elevated T2 values $(n=1)$; non-specific nonischaemic LGE $(n=1)$; slightly elevated T1 and T2 values with no pathological LGE $(n=2)$; isolated, slightly elevated T1 value $(n=1)$; and pericardial involvement $(n=1)$. All athletes with definite $(n=2)$ or possible $(n=5)$ myocardial or pericardial alterations were referred to CMR examination based on the clinical suspicion of myocardial involvement as detailed in table 2. HsTnT recorded in our institute was elevated in $4.5 \%$ of the cases $(n=6 / 133)$; among these patients, only one had myocardial alteration on CMR.

We found hinge point fibrosis in 32\% $(n=44)$ of the athletes after SARS-CoV-2 infection, which we reported as nonpathological. Fifteen healthy control athletes received contrast material. The proportion of hinge point fibrosis was similar in athletes after SARS-CoV-2 infection (44/139, 32\%) and healthy control athletes $(6 / 15,40 \% ; \mathrm{p}=0.513)$.

Table 1 shows the comparison between highly trained athletes with prior SARS-CoV-2 infection, healthy athletic controls and healthy less active controls. We found elevated cardiac volumes and myocardial mass in athletes relative to less active controls, signifying normal sport adaptation. There were no differences between the matched athletic groups regarding their LV and RV functional and volumetric parameters. LV analysis showed subtle functional alterations between athletes and controls, with the former showing slightly lower strain values. There was no difference regarding any strain parameters between athletes after SARS-CoV-2 infection and healthy control athletes. Native T1 values were slightly lower in the athletes after SARS-CoV-2 infection than in the controls, but there was no difference between athletic groups. The T2 values were not different among the three groups.

We explored the associations of native T1 and T2 mapping values with the time since confirmation of SARS-CoV-2 infection (figure 2). We did not find a correlation between T1 values and time since the infection, while T2 values showed a weak negative correlation (Rho: $-0.22, p=0.009$ ) with this parameter.

Comparison of native T1 mapping values between sexes revealed that men (median 953, IQR 934-965 ms) had significantly lower T1 values than women (median 977, IQR 959-987 $\mathrm{ms})$, regardless of whether they were healthy controls or athletes after SARS-CoV-2 infection $(\mathrm{p}<0.0001)$ (online supplemental file 1).

Fourteen elite athletes had previously undergone CMR imaging in our institute prior to obtaining positive SARS-CoV-2 PCR results (table 3). The two CMR scans for this group were performed an average of 384 days apart. Comparing examinations before and after the infection revealed no differences regarding any CMR parameters, as shown in table 3.

We compared athletes with prior SARS-CoV-2 infection regarding their symptoms (figure 3), which showed that athletes with moderate symptoms, mainly chest pain and dyspnoea, had slightly elevated native $\mathrm{T} 1$ values relative to their asymptomatic and mildly symptomatic counterparts $(p<0.05)$. However, the T1 value remained below the cut-off point for the majority of patients. Furthermore, there was no difference in the LV ejection fraction or GLS values among these groups.

We obtained follow-up in $122(83 \%)$ athletes after SARSCoV-2 infection at a median of 232 days after the infection. All but two athletes could return to sports activity safely. One of them did not return to sports due to the progression of his depression, and he currently receives medication. The other athlete experienced long-COVID syndrome, including lightheadedness and long-term rapid increase in his heartbeat. At the time of our follow-up, this athlete had a negative exercise test and was advised to restart sports activity. The outcomes of the seven athletes with CMR alteration are shown in table 2.

Online supplemental file 2 shows the acute and follow-up CMR scans in those patients with myocardial alteration $(n=4)$ who returned for a follow-up scan. In one athlete with LGE showing a non-ischaemic pattern consistent with previous myocarditis, the follow-up CMR showed slightly elevated systolic function and the shrinkage of the LGE. Among the three patients presenting with mild, isolated mapping elevation, the follow-up scan revealed that the elevated mapping values had subsided for two patients and remained slightly elevated for the last. Three athletes asked to postpone their follow-up scans due to their lack of symptoms and their ongoing sports season.

Overall, 10 athletes reported a subjectively long recovery from COVID-19. Three additional athletes said that, although they returned to sports activity, they did not reach their peak potential at the time of their follow-up. It was due to anxiety in one case and two athletes experienced mild, long-term sinus tachycardia with no apparent structural alteration. None of the national team members $(n=71)$ reported significant setbacks in their performance. In all patients who reported reinfection confirmed by PCR ( $n=4)$, we performed follow-up CMR without definite alteration (online supplemental file 3 ).

\section{DISCUSSION}

The current study presents a comprehensive analysis of the CMR findings of 147 highly trained athletes following SARS-CoV-2 infection and compares them to sex-matched and age-matched healthy athletes and less active controls. In this group, where all athletes were referred to the examination by a cardiologist, CMR revealed no overall differences regarding any volumetric, functional or tissue characteristics between athletes with prior SARS-CoV-2 infection and matched healthy athletes. However, a minority of the athletes had definite $(n=2,1.4 \%)$ or possible $(n=5,3.4 \%)$ myocardial or pericardial alterations on CMR. Four of these athletes were moderately symptomatic; two of them had long COVID; and one had mild symptoms. 


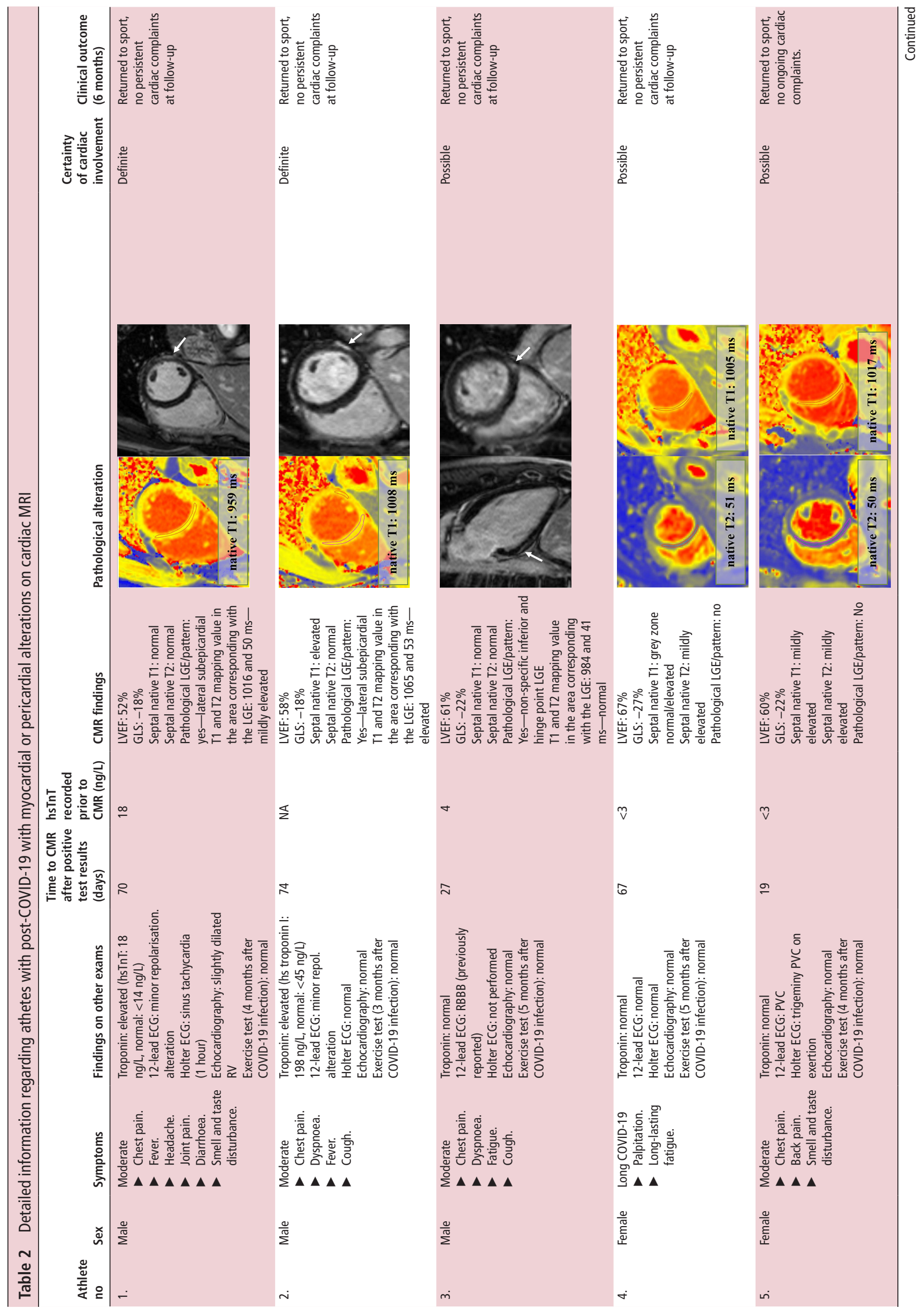



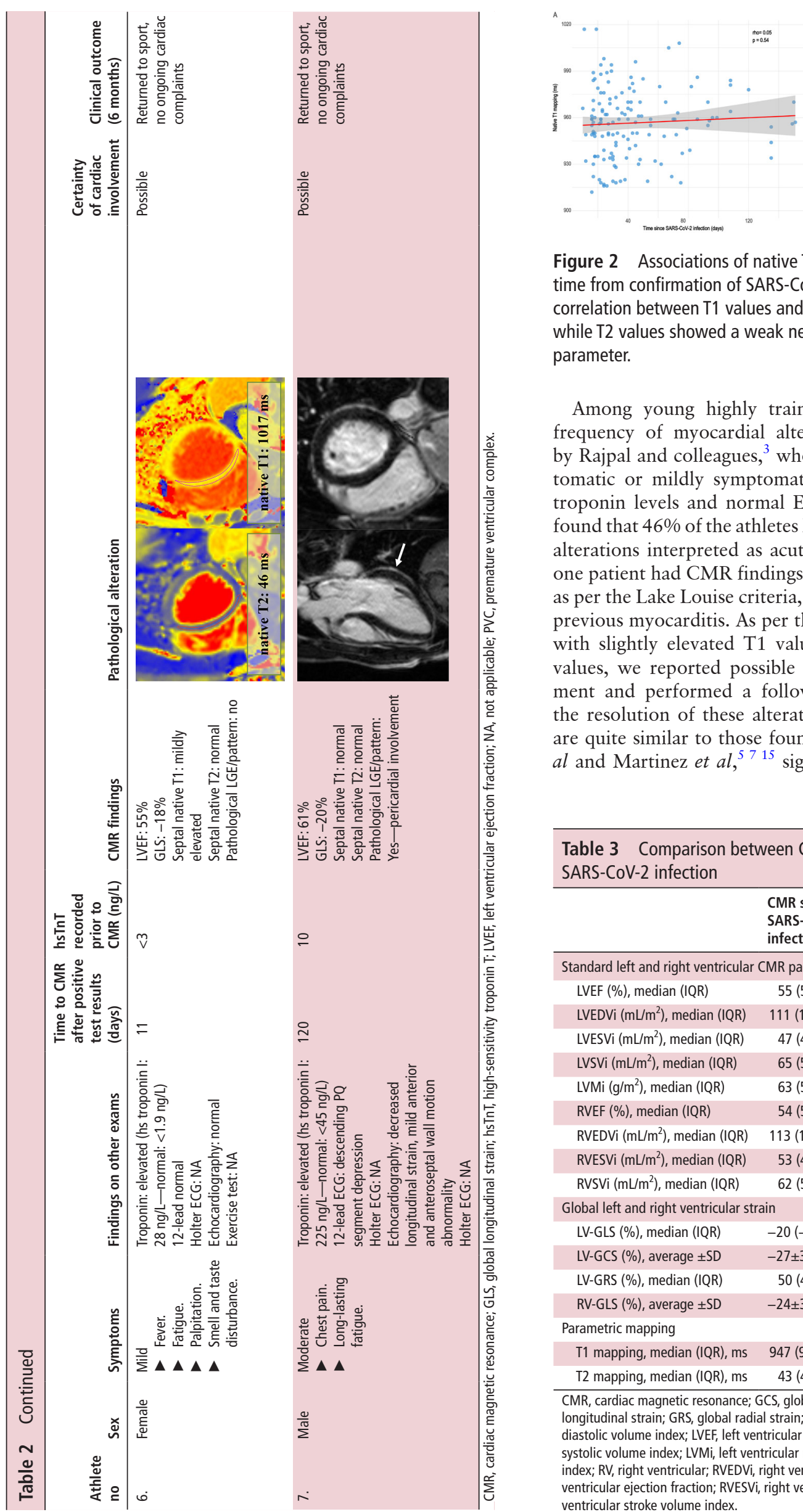

Figure 2 Associations of native T1 and T2 mapping values and the time from confirmation of SARS-CoV-2 infection. We did not find a correlation between $\mathrm{T} 1$ values and time since SARS-CoV-2 infection, while T2 values showed a weak negative correlation with this parameter.

Among young highly trained athletes, we found a lower frequency of myocardial alteration than previously reported by Rajpal and colleagues, ${ }^{3}$ who performed CMR for 26 asymptomatic or mildly symptomatic collage athletes with negative troponin levels and normal ECG and echocardiography. They found that $46 \%$ of the athletes had LGE and $15 \%$ had myocardial alterations interpreted as acute myocarditis. In our study, only one patient had CMR findings consistent with acute myocarditis as per the Lake Louise criteria, ${ }^{14}$ and one had findings suggesting previous myocarditis. As per those three athletes who presented with slightly elevated T1 values with or without elevated T2 values, we reported possible mild diffuse myocardial involvement and performed a follow-up CMR scan, which showed the resolution of these alterations in two patients. Our results are quite similar to those found by Starekova et al, Moulson et $a l$ and Martinez et $a l,{ }^{5715}$ signifying the modest prevalence of

Table 3 Comparison between CMR examinations before and after SARS-CoV-2 infection

\begin{tabular}{|c|c|c|c|}
\hline & $\begin{array}{l}\text { CMR scan before } \\
\text { SARS-CoV-2 } \\
\text { infection }(n=14)\end{array}$ & $\begin{array}{l}\text { CMR scan after } \\
\text { SARS-CoV-2 } \\
\text { infection }(n=14)\end{array}$ & $P$ values \\
\hline \multicolumn{4}{|c|}{ Standard left and right ventricular CMR parameters } \\
\hline LVEF (\%), median (IQR) & $55(53-58)$ & $57(53-61)$ & 0.091 \\
\hline LVEDVi $\left(\mathrm{mL} / \mathrm{m}^{2}\right)$, median (IQR) & $111(103-120)$ & $117(104-125)$ & 0.305 \\
\hline LVESVi (mL/m²), median (IQR) & $47(46-59)$ & $51(42-55)$ & 0.216 \\
\hline LVSVi (mL/m²), median (IQR) & $65(57-67)$ & $65(60-75)$ & 0.135 \\
\hline LVMi $\left(\mathrm{g} / \mathrm{m}^{2}\right)$, median (IQR) & $63(59-77)$ & $70(62-82)$ & 0.502 \\
\hline RVEF (\%), median (IQR) & $54(52-56)$ & $57(53-60)$ & 0.091 \\
\hline RVEDVi $\left(\mathrm{mL} / \mathrm{m}^{2}\right)$, median (IQR) & $113(107-120)$ & $116(100-122)$ & 0.946 \\
\hline RVESVi $\left(\mathrm{mL} / \mathrm{m}^{2}\right)$, median (IQR) & $53(44-60)$ & $49(45-57)$ & 0.094 \\
\hline RVSVi $\left(\mathrm{mL} / \mathrm{m}^{2}\right)$, median (IQR) & $62(57-69)$ & $64(59-73)$ & 0.38 \\
\hline \multicolumn{4}{|l|}{ Global left and right ventricular strain } \\
\hline LV-GLS (\%), median (IQR) & $-20(-22$ to -19$)$ & $-20(-21$ to -18$)$ & 0.241 \\
\hline LV-GCS $(\%)$, average \pm SD & $-27 \pm 3$ & $-28 \pm 5$ & 0.883 \\
\hline LV-GRS (\%), median (IQR) & $50(45-55)$ & $49(45-53)$ & 0.715 \\
\hline RV-GLS $(\%)$, average \pm SD & $-24 \pm 3$ & $-23 \pm 3$ & 0.29 \\
\hline \multicolumn{4}{|l|}{ Parametric mapping } \\
\hline T1 mapping, median (IQR), ms & 947 (932-961) & 937 (933-966) & 0.791 \\
\hline T2 mapping, median (IQR), ms & $43(43-45)$ & $44(42-46)$ & 0.32 \\
\hline
\end{tabular}

CMR, cardiac magnetic resonance; GCS, global circumferential strain; GLS, global longitudinal strain; GRS, global radial strain; LV, left ventricular; LVEDVi, left ventricular end diastolic volume index; LVEF, left ventricular ejection fraction; LVESVi, left ventricular end systolic volume index; LVMi, left ventricular mass index; LVSVi, left ventricular stroke volume index; RV, right ventricular; RVEDVi, right ventricular end diastolic volume index; RVEF, right ventricular ejection fraction; RVESVi, right ventricular end systolic volume index; RVSVi, right ventricular stroke volume index. 


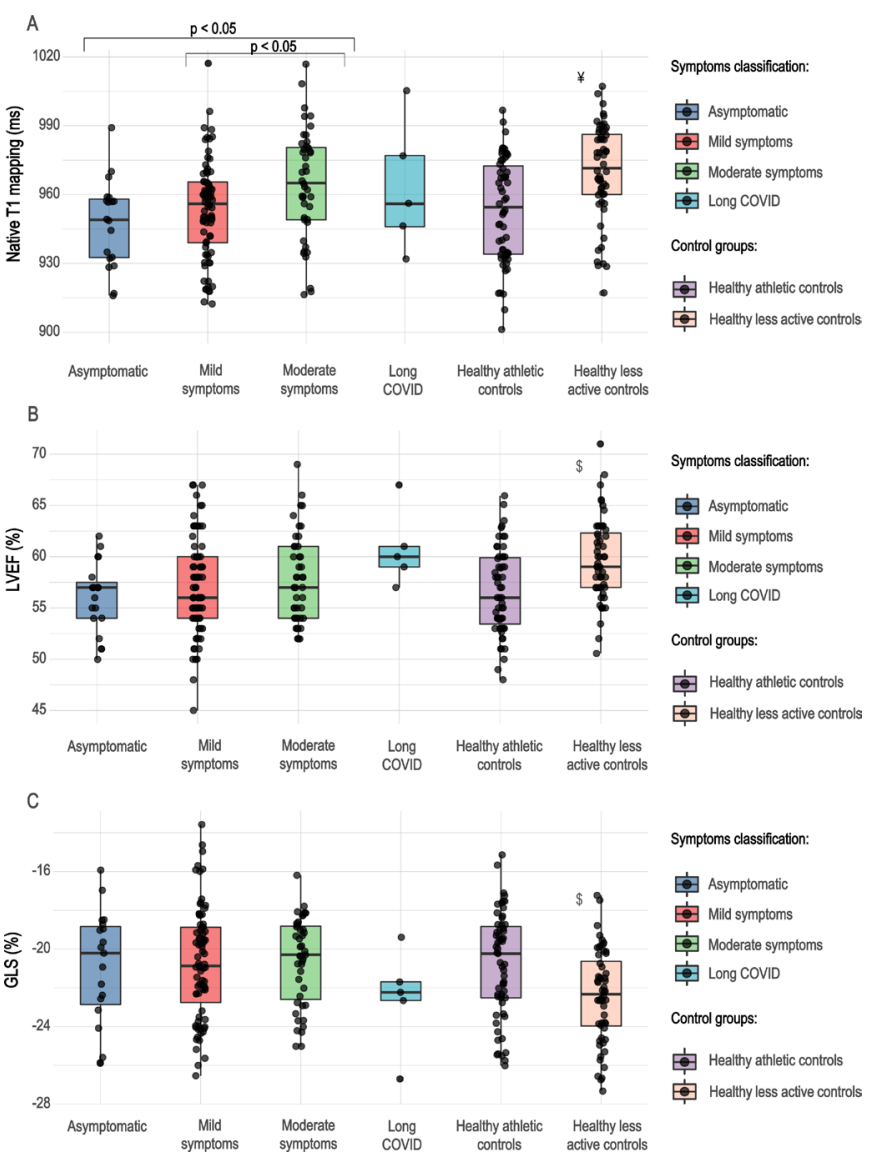

Figure 3 Boxplots of native T1 mapping, LVEF and GLS values by symptom group. Moderately symptomatic athletes with post-COVID-19 had elevated native $\mathrm{T} 1$ values relative to asymptomatic and mildly symptomatic infections $(p<0.05)$. However, the T1 value remained below the normal cut-off point for the majority of patients. There was no difference in the LVEF or GLS values among these groups. $¥$, KruskalWallis test showing a significant difference between healthy, less active controls and asymptomatic and mildly symptomatic athletes after SARSCoV-2 infection and healthy athletic controls; \$, Kruskal-Wallis test showing a significant difference between healthy, less active controls and asymptomatic, mildly and moderately symptomatic athletes after SARS-CoV-2 infection, and healthy athletic controls. GLS, global longitudinal strain; LVEF, left ventricular ejection fraction.

myocardial involvement after SARS-CoV-2 in young, otherwise healthy individuals. In a nationwide research study among US collegiate athletes conducted by Moulson and colleagues, they also found the cardiac involvement among athletes as low as $0.7 \%$, and interestingly, they found that CMR scans performed on the basis of clinical symptoms were four times more likely to show myocardial alterations as opposed to those that were performed as a primary screening method. ${ }^{7}$ Overall, these findings are in line with pathological reports showing that only $1 \%-7 \%$ of 277 autopsied hearts across 22 publications had COVID-19-related myocarditis according to histopathological findings, ${ }^{16}$ although in a different patient population. In our cohort, only one athlete presented with pericardial involvement; this finding is in contrast with the case series of Brito and colleagues, who found pericardial enhancement in $39.5 \%$ of athletes. ${ }^{4}$

We did not find a difference regarding the proportion of hinge point fibrosis after SARS-CoV-2 infection in athletes and healthy control athletes; however, only a relatively small number of control athletes received contrast material $(n=15)$. We found a slightly higher proportion of hinge point fibrosis than Clark et al (athletes after SARS-CoV-2 infection) and a lower ratio than Domenech-Ximenos et al in endurance athletes before the pandemic (32\% vs $22 \%$ vs $38 \%$ ). Of note, these athletic groups were different from ours in some respects, including the ratio of female athletes (36\% vs $63 \%$ vs $47 \%$ ), sports discipline and training hours, which might account for the differences. ${ }^{6} 17$

Our findings regarding sport adaptation are in line with the current literature. ${ }^{18} 19$ Data are scarce regarding the featuretracking strain analysis of highly trained athletes, and the tendencies described in our study (slightly lower global strain values among highly trained men) are similar to those in the currently available publications using echocardiography. ${ }^{20-22}$ Comparing athletes with prior SARS-CoV-2 infection and matched athletes showed no difference between CMR parameters, including strain parameters and native T1 and T2 mapping values. This finding confirms the results of the recent research letter by Clark et $a l,{ }^{6}$ who reported only a small difference between athletes post-COVID-19 and healthy control athletes regarding their mid-septal T2 mapping values. However, the groups in their study were matched by training load, not age or sex, which could have contributed to differences. While the cohort study by Puntmann et $a l^{1}$ reported a higher prevalence of findings, new studies have shown similar results to ours, although in very different populations. ${ }^{2324} \mathrm{McDiarmid}$ et $a l^{25}$ previously demonstrated that physiological hypertrophy slightly decreased the T1 value among highly trained athletes. We also found that, similar to other CMR parameters, men and women have distinct native T1 and T2 values, which justifies the use of sex-matched control groups when interpreting mapping alterations.

We did not find a correlation between T1 mapping values and the time passed since SARS-CoV-2 infection, similar to what Knight $e \mathrm{al}^{24}$ found in their study with a somewhat longer delay between SARS-CoV-2 infection and CMR examination (median 68 vs 32 days). A weak but significant correlation was found between T2 mapping and time since infection. This might suggest a reduction in subclinical oedema over time; however, we need more information to confirm this finding.

One unique strength of this study is that 14 athletes had undergone a previous CMR scan at our institute with a standardised protocol; therefore, we were able to compare the results of the two scans. This comparison, however, showed no differences between CMR parameters before and after SARSCoV-2 infection.

Follow-up at median 232 days after COVID-19 infections showed the majority of athletes returned to high levels of sports activity $(n=120 / 122)$, although some could not reach their peak performance $(n=3)$ and some experienced reinfection $(n=4)$.

The comparison between athletes with different symptoms revealed slightly elevated T1 mapping values among athletes with chest complaints relative to asymptomatic and mildly symptomatic athletes; however, this did not lead to a reduction in systolic heart function. Moreover, T1 values remained in the normal range for most patients. Currently, there are no data regarding the subclinical cardiac alterations caused by mild forms of systemic viral infections such as influenza and whether they are detectable on CMR. We believe that studies investigating the long-term impact of isolated T1 and T2 mapping elevations are necessary to understand the exact prognostic significance of these alterations, and in this study, we share the concerns of Moulson and Baggish ${ }^{26}$ regarding the use of these highly sensitive, although less well-understood techniques, in the screening of otherwise healthy athletes with prior SARS-CoV-2 infection. 
The current consensus document ${ }^{9}$ regarding the use of CMR in athletes after SARS-CoV-2 infection highlights the importance of well-established screening methods such as troponin, ECG and echocardiography. Moreover, in suspected arrhythmias, further examinations such as 24-hour Holter monitoring might be beneficial, ${ }^{89}$ and premature ventricular beats on exercise test might suggest scar on CMR examination as demonstrated by recent studies, enabling a better targeting of CMR scans. ${ }^{27}$ In agreement with this, our results caution against the routine use of CMR for troponin-negative, asymptomatic, or mildly symptomatic patients with COVID-19, as it may lead to false conclusions.

\section{LIMITATIONS}

This was a single-centre study performed in a major CMR referral centre. Approximately one-third of the athletes after SARS-CoV-2 infection were referred from other institutions; therefore, their clinical data were provided by the referring clinicians. All athletes included in our study were Caucasian and experienced asymptomatic, mild/moderate or long COVID-19; thus, our conclusions are only applicable to this specific group. Because our study included patients referred by a cardiologist, the reported prevalence of abnormal CMR findings may be overestimated compared with a non-selected population of athletes with SARS-CoV-2 infection. In addition, the clinical implications of CMR abnormalities in the absence of cardiovascular symptoms remains unknown. Lastly, only a proportion of healthy control athletes received contrast agent during their CMR; thus, findings related to LGE in the athletic control group could have been missed.

\section{What are the new findings?}

- Among 147 highly trained athletes after SARS-CoV-2 infection, we found that cardiac involvement on cardiac magnetic resonance (CMR) was present in only seven $(4.8 \%)$ patients, among whom only two (1.4\%) presented with definite signs of myocarditis.

- Comparing athletes after SARS-CoV-2 infection and healthy sex-matched and age-matched athletes showed no difference between CMR parameters, including strain and native $\mathrm{T} 1$ and T2 mapping values.

- Comparison between CMR examinations before and after the infection $(n=14)$ revealed no differences regarding any CMR parameters.

- Follow-up at a median of 232 days after the infections showed the majority of athletes returned to high levels of sports activity ( $n=120 / 122,98.4 \%)$.

\section{How might it impact on clinical practice in the future?}

- Cardiac involvement has a low prevalence among highly trained athletes after SARS-CoV-2 infection.

- Matched control groups are essential for the interpretation of isolated T1 or T2 mapping alterations.

- Our results caution against the routine use of CMR for troponin-negative, asymptomatic or mildly symptomatic patients after SARS-CoV-2 infection.

\section{CONCLUSION}

Among 147 consecutively included highly trained athletes after SARS-CoV-2 infection and referred by a cardiologist, we found cardiac involvement in $4.7 \%$ using CMR, among whom only two $(1.4 \%)$ presented with definite signs of myocarditis. Our results suggest that cardiac involvement occurs with modest frequency among asymptomatic and mildly/moderately symptomatic SARS-CoV-2 infections in young athletes. Comparisons between athletes after SARS-CoV-2 infection and matched healthy athletes showed no difference between CMR parameters, including strain parameters and T1 and T2 mapping values. Moreover, there was no difference in CMR parameters among athletes examined before and after the infection. The follow-up revealed that the majority of athletes returned to high levels of sports activity without any persisting symptoms.

\section{Twitter Liliána Szabó @|iliana_e_szabo}

Acknowledgements We acknowledge the invaluable technical support from Mate Kiss, Attila Toth and Csilla Czimbalmos. We thank the dedicated work of the Semmelweis University Hear and Vascular Centre radiography team, especially Eva Lehotai and Piroska Bajorne. Contributors were not compensated for their work.

Contributors LS contributed to the conception of the study, investigation, analysis, interpretation of the data and writing the original draft. VJ, CF, OK, NS and EC contributed to the formal analysis of the data, investigation, and project administration. ZD, AK, BL, HK and FIS contributed to the investigation and methodology of the study and edited the manuscript. DB and BM contributed to the conceptualisation, interpretation of the data, funding acquisition, supervision and editing of the manuscript. HV contributed to the conceptualisation, investigation, interpretation of the data, funding acquisition, supervision, writing and editing of the manuscript. All authors gave their final approval to the manuscript. LS and HV are responsible for the overall content as guarantors.

Funding The research was financed by the Thematic Excellence Programme (Tématerületi Kiválósági Program, 2020-4.1.1.-TKP2020) of the Ministry for Innovation and Technology in Hungary, within the framework the Bioimaging Thematic Programme of Semmelweis University and by the Ministry of Innovation and Technology NRDI Office within the framework of the Artificial Intelligence National Laboratory Programme. This project was supported by a grant from the National Research, Development and Innovation Office (NKFIH) of Hungary (K135076 to BM). This project was supported by a grant from the NKFIH of Hungary (2020-1.1.6-JÖVŐ-2021-00013). LS was supported by the ÚNKP-20-3-II-SE-61 New National Excellence Programme of the Ministry for Innovation and Technology from the source of the National Research, Development and Innovation Fund and the Development of Scientific Workshops of Medical, Health Sciences and Pharmaceutical Education project (project identification number: EFOP-3.6.3-VEKOP16-2017-00009).

\section{Competing interests None declared.}

Patient consent for publication Not applicable.

Ethics approval Ethical approval was obtained from the National Public Health Center in accordance with the ethical standards laid out in the 1964 Declaration of Helsinki and its later amendments. IV/9697-1/2020/EKU. All participants gave their written informed consent. It was not possible to involve patients in the design or reporting of our current study. Athletes with post-COVID-19 are currently involved in the 'return-to-play practices' and dissemination plans of our ongoing research.

Provenance and peer review Not commissioned; externally peer reviewed.

Data availability statement Data are available upon reasonable request. All data are available upon reasonable request.

Supplemental material This content has been supplied by the author(s). It has not been vetted by BMJ Publishing Group Limited (BMJ) and may not have been peer-reviewed. Any opinions or recommendations discussed are solely those of the author(s) and are not endorsed by BMJ. BMJ disclaims all liability and responsibility arising from any reliance placed on the content. Where the content includes any translated material, BMJ does not warrant the accuracy and reliability of the translations (including but not limited to local regulations, clinical guidelines, terminology, drug names and drug dosages), and is not responsible for any error and/or omissions arising from translation and adaptation or otherwise.

Open access This is an open access article distributed in accordance with the Creative Commons Attribution 4.0 Unported (CC BY 4.0) license, which permits others to copy, redistribute, remix, transform and build upon this work for any 
purpose, provided the original work is properly cited, a link to the licence is given, and indication of whether changes were made. See: https://creativecommons.org/ licenses/by/4.0/.

\section{ORCID iDs}

Liliána Szabó http://orcid.org/0000-0002-4699-3648

Vencel Juhász http://orcid.org/0000-0001-9100-4952

Attila Kovács http://orcid.org/0000-0003-2320-6434

Bálint Károly Lakatos http://orcid.org/0000-0002-7627-5620

Hajnalka Vágó http://orcid.org/0000-0002-3568-3572

\section{REFERENCES}

1 Puntmann VO, Carerj ML, Wieters I, et al. Outcomes of cardiovascular magnetic resonance imaging in patients recently recovered from coronavirus disease 2019 (COVID-19). JAMA Cardiol 2020;5:1265-73.

2 Leiner T, Bogaert J, Friedrich MG, et al. SCMR position paper (2020) on clinical indications for cardiovascular magnetic resonance. J Cardiovasc Magn Reson 2020;22:76.

3 Rajpal S, Tong MS, Borchers J, et al. Cardiovascular magnetic resonance findings in competitive athletes recovering from COVID-19 infection. JAMA Cardiol 2020;132:10-12.

4 Brito D, Meester S, Yanamala N, et al. High prevalence of pericardial involvement in college student athletes recovering from COVID-19. JACC Cardiovasc Imaging 2021;14:541-55.

5 Starekova J, Bluemke DA, Bradham WS, et al. Evaluation for myocarditis in competitive student athletes recovering from coronavirus disease 2019 with cardiac magnetic resonance imaging. JAMA Cardio/ 2021;6:945-6.

6 Clark DE, Parikh A, Dendy JM, et al. COVID-19 myocardial pathology evaluation in athletes with cardiac magnetic resonance (compete CMR). Circulation 2021;143:609-12.

7 Moulson N, Petek BJ, Drezner JA, et al. SARS-CoV-2 cardiac involvement in young competitive athletes. Circulation 2021;144:256-66.

8 Phelan D, Kim JH, Elliott MD, et al. Screening of potential cardiac involvement in competitive athletes recovering from COVID-19: an expert consensus statement. JACC Cardiovasc Imaging 2020;13:2635-52.

9 Kim JH, Levine BD, Phelan D. Coronavirus disease 2019 and the athletic heart: emerging perspectives on pathology, risks, and return to play. JAMA Cardiol 2020:1-9.

10 NIH. Coronavirus disease 2019 (COVID-19) treatment guidelines. Nih2020;2019:130

11 Vago H, Szabo L, Dohy Z, et al. Cardiac magnetic resonance findings in patients recovered from COVID-19: initial experiences in elite athletes. JACC CardiovasC Imaging 2021;14:2-3.
12 Pelliccia A, Sharma S, Gati S, et al. 2020 ESC guidelines on sports cardiology and exercise in patients with cardiovascular disease. Eur Heart J 2021;42:17-96.

13 Schulz-Menger J, Bluemke DA, Bremerich J. Standardized image interpretation and post-processing in cardiovascular magnetic resonance - 2020 update: Society for Cardiovascular Magnetic Resonance (SCMR): Board of Trustees Task Force on Standardized Post-Processing. J Cardiovasc Magn Reson 2020;22:1-22.

14 Ferreira VM, Schulz-Menger J, Holmvang G, et al. Cardiovascular Magnetic Resonance in Nonischemic Myocardial Inflammation: Expert Recommendations. J Am Coll Cardiol 2018;72:3158-76

15 Martinez MW, Tucker AM, Bloom OJ, et al. Prevalence of inflammatory heart disease among professional athletes with prior COVID-19 infection who received systematic Return-to-Play cardiac screening. JAMA Cardiol 2021;6:745-8.

16 Halushka MK, Vander Heide RS. Myocarditis is rare in COVID-19 autopsies: cardiovascular findings across 277 postmortem examinations. Cardiovasc Pathol 2021:50:107300.

17 Domenech-Ximenos B, Sanz-de la Garza M, Prat-González S, et al. Prevalence and pattern of cardiovascular magnetic resonance late gadolinium enhancement in highly trained endurance athletes. J Cardiovasc Magn Reson 2020;22:62.

18 Petersen SE, Hudsmith LE, Robson MD, et al. Sex-Specific characteristics of cardiac function, geometry, and mass in young adult elite athletes. J Magn Reson Imaging 2006;24:297-303.

19 Csecs I, Czimbalmos C, Toth A, et al. The impact of sex, age and training on biventricular cardiac adaptation in healthy adult and adolescent athletes: cardiac magnetic resonance imaging study. Eur J Prev Cardiol 2020;27:540-9.

20 Caselli S, Montesanti D, Autore C, et al. Patterns of left ventricular longitudinal strain and strain rate in Olympic athletes. J Am Soc Echocardiogr 2015:28:245-53.

21 Lakatos BK, Molnár Andrea Ágnes, Kiss 0, et al. Relationship between cardiac remodeling and exercise capacity in elite athletes: incremental value of left atrial morphology and function assessed by three-dimensional echocardiography. J Am Soc Echocardiogr 2020:33:101-9.

22 D'Andrea A, Radmilovic J, Carbone A, et al. Speckle tracking evaluation in endurance athletes: the "optimal" myocardial work. Int J Cardiovasc Imaging 2020;36:1679-88.

23 Pan C, Zhang Z, Luo L, et al. Cardiac T1 and T2mapping showed myocardial involvement in recovered COVID-19 patients initially considered devoid of cardiac damage. J Magn Reson Imaging 2021:54:421-8.

24 Knight DS, Kotecha T, Razvi Y, et al. COVID-19: myocardial injury in survivors. Circulation 2020:142:1120-2.

25 McDiarmid AK, Swoboda PP, Erhayiem B, et al. Athletic cardiac adaptation in males is a consequence of elevated myocyte mass. Circ Cardiovasc Imaging 2016;9:1-8.

26 Moulson N, Baggish A. Subclinical COVID-19 Cardiac Imaging Findings: Resurgence of the Athletic "Grey-Zone". JACC Cardiovasc Imaging 2021;14:556-8.

27 Corrado D, Drezner JA, D'Ascenzi F, et al. How to evaluate premature ventricular beats in the athlete: critical review and proposal of a diagnostic algorithm. Br J Sports Med 2020:54:1142-8 